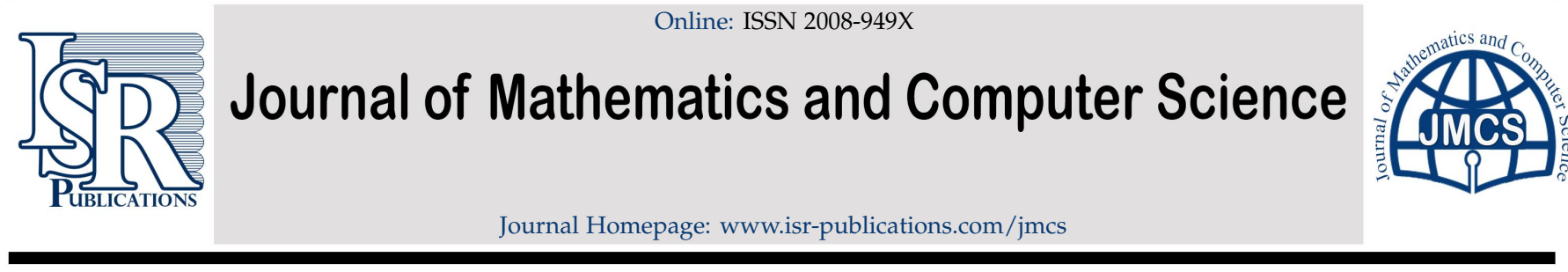

\title{
Ostrowski type integral inequalities, weighted Ostrowski, and trapezoid type integral inequalities with powers
}

\author{
Al-Taher A. Ghareeb ${ }^{a, *}$, Samir H. Saker ${ }^{b, c}$, A. A. Ragab ${ }^{d}$ \\ ${ }^{a}$ Department of Basic Science, Faculty of Engineering, Sinai University, El-Arish, Egypt. \\ ${ }^{b}$ Department of Mathematics, Faculty of Science, New Mansoura University, New Mansoura, Egypt. \\ ${ }^{c}$ Department of Mathematics, Faculty of Science, Mansoura University, Mansoura 35516, Egypt. \\ ${ }^{d}$ Department of Mathematics, Faculty of Science, Al-Azhar University, Nasr City (11884), Cairo, Egypt.
}

\begin{abstract}
In this paper, we prove some new weighted Ostrowski and Trapezoid type inequalities with powers on time scales. The results will be proved by employing the generalized version of Montgomery identity with weights on time scales designed and proved for this purpose. As special cases, we will derive some new weighted discrete inequalities of Ostrowski and Trapezoid types which to the best of the authors' knowledge are essentially new.
\end{abstract}

Keywords: Ostrowski inequality integral inequalities, Trapezoid inequality, discrete inequalities, time scales.

2020 MSC: 26D15, 34A40, 39A12, 34N05.

(C)2022 All rights reserved.

\section{Introduction}

During the recent decades the theory of inequalities has been used in investigating and studying complex problems, we refer the reader to the papers $[1-3,6,10,14,20,30,34,36-38,40,42]$ and the references cited therein. The theory of inequalities has also been used in the analysis of qualitative properties of solutions of partial differential equations (in short, PDEs); see [29]. See also [18, 19, 26] for analyses of partial differential equations whose studies rely on the employments of functional inequalities. One of these inequalities is the inequality proved by Ostrowski in [34]. In particular, Ostrowski proved that

$$
\left|f(t)-\frac{1}{b-a} \int_{a}^{b} f(s) d s\right| \leqslant\left[\frac{1}{4}+\left(\frac{t-\frac{a+b}{2}}{b-a}\right)^{2}\right](b-a) \sup _{t \in(a, b)}\left|f^{\prime}(t)\right|,
$$

where $f:[a, b] \longrightarrow \mathbb{R}$ is continuous on $[a, b], f$ and $f^{\prime}$ are differentiable on $(a, b)$. The inequality is sharp in the sense that the constant $1 / 4$ cannot be replaced by a smaller quantity. This inequality has

\footnotetext{
*Corresponding author

Email addresses: altaher_ali2008@yahoo.com (Al-Taher A. Ghareeb), shsaker@mans.edu.eg (Samir H. Saker), awaragab15852@gmail.com (A. A. Ragab)
}

doi: $10.22436 /$ jmcs.026.03.07

Received: 2021-10-04 Revised: 2021-10-17 Accepted: 2021-11-05 
powerful applications in numerical integration, probability and optimization theory, stochastic, statistics, information and integral operator theory, see for example [6, 7, 16, 23]. In 1938, Ostrowski [34] also proved the inequality

$$
\left[f(t)-\frac{1}{b-a} \int_{a}^{b} f(\eta) d \eta\right] \leqslant\left[\frac{(t-a)^{2}+(b-t)^{2}}{2(b-a)}\right] \sup _{t \in(a, b)}\left|f^{\prime}(t)\right|,
$$

by employing the Montgomery identity [31]. In 1994, Mitrinović, Pečarić and Fink [32] proved the Trapezoid inequality

$$
\left|\int_{a}^{b} f(x) d x-\frac{f(a)+f(b)}{2}(b-a)\right| \leqslant \frac{\sup _{t \in(a, b)}\left|f^{\prime \prime}(t)\right|}{12}(b-a)^{3},
$$

where $f:[a, b] \rightarrow \mathbb{R}$ is supposed to be twice differentiable on the interval $(a, b)$ with the second derivative bounded on $(a, b)$.

In the last years Ostrowski's inequality has been generalized and extended by several authors on time scales, see $[1,5-7,10,11,16,24,28,30,35,46]$ and the references cited therein. The general idea is to prove a result for dynamic inequalities where the domain of the unknown function is a so-called time scale $\mathbb{T}$, to avoid proving results twice, once for differential inequality and once again for difference inequality. This idea goes back to its founder Stefan Hilger [21] who started the study of dynamic equations on time scales. Since the integral and discrete inequalities are important in the analysis of qualitative properties of solutions of differential and difference equations, see $[15,17,25]$, we also believe that the dynamic inequalities with weights on time scales will play the same effective role in the analysis of qualitative properties of dynamic equations with boundary conditions like oscillation, nonoscillation and distribution of zeros of solutions, see $[8,9]$. For related dynamic inequalities on time scales, we refer the reader to the papers [36-45] and the books [2,3]. In [11] Bohner and Matthews generalized the Montgomery identity on time scales and proved that

$$
f(t)=\frac{1}{b-a}\left[\int_{a}^{b} f^{\sigma}(\eta) \Delta \eta+\int_{a}^{b} \Psi(t, \eta) f^{\Delta}(\eta) \Delta \eta\right],
$$

where $a, b \in \mathbb{T}$ with $a<b$ and $f \in C_{r d}^{1}\left([a, b]_{\mathbb{T}}, \mathbb{R}\right)$ and $\Psi$ is defined by

$$
\Psi(t, s):= \begin{cases}s-a, & s \in[a, t)_{\mathbb{T}} \\ s-b, & s \in[t, b]_{\mathbb{T}}\end{cases}
$$

for $s, t \in[a, b]_{\mathbb{T}}$. By using the identity (1.1) Bohner et al. [11] proved Ostrowski inequality

$$
\left[f(t)-\frac{1}{b-a} \int_{a}^{b} f^{\sigma}(\eta) \Delta \eta\right] \leqslant\left(\sup _{\eta \in(a, b)}\left|f^{\Delta}(\eta)\right|\right)\left[\frac{h_{2}(t, a)+h_{2}(t, b)}{b-a}\right],
$$

where $h_{2}(t, s)$ is defined by

$$
h_{2}(t, s)=\int_{s}^{t} h_{1}(\tau, s) \Delta \tau \text { and } h_{1}(\tau, s)=\tau-s, \text { for all } s, t \in \mathbb{T} .
$$

In 2008, Karpuz and Özkan [24] generalized Ostrowski's inequality and Montgomery's identity on arbitrary time scales by means of generalized polynomials on time scales. In 2009, Liu et al. [28] proved a generalization of the inequality (1.2) on time scales. In 2010, Liu [27] established a weighted three point inequality with a parameter for mappings of bounded variation. In 2012, Liu and Tuna [30] established some weighted dynamic Ostrowski, Trapezoid, and Grüss type inequalities based on a weighted Montgomery identity on time scales. 
Following this trend and to develop the study of dynamic inequalities on time scales, we will prove some new weighted Ostrowski and Trapezoid type inequalities with powers. To obtain the main results, we will prove a generalized version of Montgomery identity with weights. As special cases, we derive some discrete inequalities which to the best of our knowledge are essentially new. The paper is organized as follows. In Section 2, we present some basic definitions and concepts on time scales. In Section 3, we will prove the basic lemmas that will be needed in the proofs of the main results. In Section 4, first, we will prove weighted Ostrowski type inequalities on time scales with powers and second, we will prove Trapezoid type inequalities on time scales with powers and derive the special cases.

\section{Preliminaries}

In this section, we present some basic definitions and concepts concerning the delta calculus on time scales. For more details, we refer the reader to the book [12]. A time scale $\mathbb{T}$ is an arbitrary nonempty closed subset of the real numbers $\mathbb{R}$.

The forward jump operator and the backward jump operator are defined by $\sigma(t):=\inf \{s \in \mathbb{T}: s>$ $t\}$, and $\rho(t):=\sup \{s \in \mathbb{T}: s<t\}$, where $\sup \emptyset=\inf \mathbb{T}$. A point $t \in \mathbb{T}$, is said to be left-dense if $\rho(t)=t$ and $t>\inf \mathbb{T}$, is right-dense if $\sigma(t)=t$, is left-scattered if $\rho(t)<t$ and right-scattered if $\sigma(t)>t$. The set of all such rd-continuous functions is denoted by $C_{r d}(\mathbb{T})$.

A function $f: \mathbb{T} \rightarrow \mathbb{R}$ is said to be right-dense continuous (rd-continuous) provided $f$ is continuous at right-dense points and at left-dense points in $\mathbb{T}$, left hand limits exist and are finite. Without loss of generality, we assume that $\sup \mathbb{T}=\infty$, and define the time scale interval $[a, b]_{\mathbb{T}}$ by $[a, b]_{\mathbb{T}}:=[a, b] \cap \mathbb{T}$.

For a function $f: \mathbb{T} \rightarrow \mathbb{R}$, we define the derivative $f^{\Delta}(t)$ to be the number, if it exists, such that for all $\epsilon>0$, there is a neighborhood $U$ of $t$ such that for all $s \in U$,

$$
\left|f(\sigma(t))-f(s)-f^{\Delta}(t)(\sigma(t)-s)\right| \leqslant \epsilon|\sigma(t)-s| .
$$

We say that $f$ is delta differentiable on $\mathbb{T}$ provided that $f^{\Delta}(t)$ exists for all $t \in \mathbb{T}$. The set of functions that are differentiable and whose derivative is rd-continuous is denoted by $C_{r d}^{1}(\mathbb{T})=C_{r d}^{1}(\mathbb{T}, \mathbb{R})$. The graininess function $\mu$ for a time scale $\mathbb{T}$ is defined by $\mu(t):=\sigma(t)-t$, and for any function $f: \mathbb{T} \rightarrow \mathbb{R}$ the notation $f^{\sigma}(t)$ denotes $f(\sigma(t))$.

Recall the following product and quotient rules for the derivative of the product $\mathrm{fg}$ and the quotient $\mathrm{f} / \mathrm{g}$ (where $\mathrm{gg}^{\sigma} \neq 0$, here $\mathrm{g}^{\sigma}=\mathrm{g} \circ \sigma$ ) of two differentiable functions $\mathrm{f}$ and $\mathrm{g}$

$$
(f g)^{\Delta}=f^{\Delta} g+f^{\sigma} g^{\Delta}=f g^{\Delta}+f^{\Delta} g^{\sigma} \text { and }\left(\frac{f}{g}\right)^{\Delta}=\frac{f^{\Delta} g-f g^{\Delta}}{g g^{\sigma}} .
$$

Let $f: \mathbb{R} \rightarrow \mathbb{R}$ be continuously differentiable and suppose $g: \mathbb{T} \rightarrow \mathbb{R}$ is delta differentiable, then $\mathrm{f} \circ \mathrm{g}: \mathbb{T} \rightarrow \mathbb{R}$ is delta differentiable and

$$
f^{\Delta}(g(t))=f^{\prime}(g(d)) g^{\Delta}(t), \quad \text { for } \quad d \in[t, \sigma(t)] .
$$

If $F^{\Delta}(t)=f(t)$, then the Cauchy (delta) integral of $f$ is defined by $\int_{t_{0}}^{t} f(s) \Delta s:=F(t)-F\left(t_{0}\right)$. It can be shown (see [12]) that if $f \in C_{r_{d}}(\mathbb{T})$, then the Cauchy integral $F(t):=\int_{t_{0}}^{t} f(s) \Delta s$ exists, where $t_{0} \in \mathbb{T}$, and satisfies $F^{\Delta}(t)=f(t)$, for $t \in \mathbb{T}$. An infinite integral is defined as $\int_{a}^{\infty} f(t) \Delta t=\lim _{b \rightarrow \infty} \int_{a}^{b} f(t) \Delta t$. Integration on discrete time scales is defined by

$$
\int_{a}^{b} f(t) \Delta t=\sum_{t \in[a, b)} \mu(t) f(t) .
$$

The integration by parts on time scales reads

$$
\int_{a}^{b} u(t) v^{\Delta}(t) \Delta t=[u(t) v(t)]_{a}^{b}-\int_{a}^{b} u^{\Delta}(t) v^{\sigma}(t) \Delta t
$$


Now, we define the Taylor monomials defined in [13]. These types of monomials are important because they are intimately related to Cauchy functions for certain dynamic equations which are important in variations of constants formulas.

The Taylor monomials $h_{k}: \mathbb{T} \times \mathbb{T} \rightarrow \mathbb{R}, k \in \mathbb{N}_{0}=\mathbb{N} \cup\{0\}$, are defined recursively as follows. The function $h_{0}$ is defined by $h_{0}(t, s)=1$, for all $s, t \in \mathbb{T}$, and given $h_{k}$ for $k \in \mathbb{N}_{0}$, the function $h_{k+1}$ is defined by

$$
h_{k+1}(t, s)=\int_{s}^{t} h_{k}(\tau, s) \Delta \tau, \text { for all } s, t \in \mathbb{T} .
$$

If we let $h_{k}^{\Delta}(t, s)$ denote for each fixed $s \in \mathbb{T}$, the derivative of $h(t, s)$ with respect to $t$, then

$$
h_{k}^{\Delta}(t, s)=h_{k-1}(t, s), \quad k \in \mathbb{N}, t \in \mathbb{T},
$$

for each fixed $s \in \mathbb{T}$.

The above definition obviously implies $h_{1}(t, s)=t-s$, for all $s, t \in \mathbb{T}$. In the case when $\mathbb{T}=\mathbb{R}$, then $\sigma(t)=t, \mu(t)=0, y^{\Delta}(t)=y^{\prime}(t)$, and

$$
h_{k}(t, s)=\frac{(t-s)^{k}}{k !}, \text { for all } s, t \in \mathbb{R} .
$$

\section{Basic lemmas}

In this section, we prove the basic lemmas that will be needed in the proofs of the main results. To do this, we need the following inequalities (see [4, Page 51])

$$
\begin{aligned}
& a^{\lambda}+b^{\lambda} \leqslant(a+b)^{\lambda} \leqslant 2^{\lambda-1}\left(a^{\lambda}+b^{\lambda}\right), \text { if } a, b \geqslant 0, \lambda \geqslant 1, \\
& a^{\lambda}+b^{\lambda} \geqslant(a+b)^{\lambda} \geqslant 2^{\lambda-1}\left(a^{\lambda}+b^{\lambda}\right), \text { if } a, b \geqslant 0,0 \leqslant \lambda \leqslant 1,
\end{aligned}
$$

and

$$
a^{\lambda}+b^{\lambda} \geqslant 2^{1-\lambda}(a+b)^{\lambda}, \text { if } a, b>0, \lambda<0 .
$$

Lemma 3.1. Let $\mathrm{a}, \mathrm{b} \in \mathbb{T}$ with $\mathrm{a}<\mathrm{b}, 0 \leqslant \mathrm{k} \leqslant 1, w:[\mathrm{a}, \mathrm{b}]_{\mathbb{T}} \rightarrow[0, \infty)$ be rd-continuous and positive function and $\mathrm{f}, \mathrm{h}:[\mathrm{a}, \mathrm{b}]_{\mathbb{T}} \rightarrow \mathbb{R}$ are $\Delta$-differentiable functions such that $\mathrm{h}^{\Delta}(\mathrm{t})=w(\mathrm{t})$ on $[\mathrm{a}, \mathrm{b}]_{\mathbb{T}}$. Define

$$
s(x, t)= \begin{cases}h(t)-((1-k) h(a)+k h(x)), & a \leqslant t<x \\ h(t)-(k h(x)+(1-k) h(b)), & x \leqslant t \leqslant b\end{cases}
$$

Then for all $\mathrm{x} \in[\mathrm{a}, \mathrm{b}]_{\mathbb{T}}$, we have

$$
\begin{aligned}
\int_{a}^{b} s^{p}(x, t) f^{\Delta}(t) \Delta t \leqslant & \left\{\beta(1-k)^{p} f(x)+k^{p}\left[\left(\frac{\int_{a}^{x} w(t) \Delta t}{\int_{a}^{b} w(t) \Delta t}\right)^{p} f(a)+\left(\frac{\int_{x}^{b} w(t) \Delta t}{\int_{a}^{b} w(t) \Delta t}\right)^{p} f(b)\right]\right\} \\
& \times\left(\int_{a}^{b} w(t) \Delta t\right)^{p}-p\left[\int_{a}^{x}[h(\sigma(t))-((1-k) h(a)+k h(x))]^{p-1} w(t) f(\sigma(t)) \Delta t\right. \\
& \left.+\int_{x}^{b}[h(\sigma(t))-(k h(x)+(1-k) h(b))]^{p-1} w(t) f(\sigma(t)) \Delta t\right]
\end{aligned}
$$

where

$$
\beta= \begin{cases}1, & \text { if } p \geqslant 1 \\ 2^{1-p}, & \text { if } 0 \leqslant p \leqslant 1\end{cases}
$$


Proof. By using integration by parts (2.3), we see that

$$
\begin{aligned}
& \int_{a}^{b} s^{p}(x, t) f^{\Delta}(t) \Delta t= \int_{a}^{x} s^{p}(x, t) f^{\Delta}(t) \Delta t+\int_{x}^{b} s^{p}(x, t) f^{\Delta}(t) \Delta t \\
&= \int_{a}^{x}[h(t)-((1-k) h(a)+k h(x))]^{p} f^{\Delta}(t) \Delta t \\
&+\int_{x}^{b}[h(t)-(k h(x)+(1-k) h(b))]^{p} f^{\Delta}(t) \Delta t \\
&= {[h(x)-((1-k) h(a)+k h(x))]^{p} f(x)-[h(a)-((1-k) h(a)+k h(x))]^{p} f(a) } \\
&-\int_{a}^{x}\left([h(t)-((1-k) h(a)+k h(x))]^{p}\right)^{\Delta} f(\sigma(t)) \Delta t \\
&+[h(b)-(k h(x)+(1-k) h(b))]^{p} f(b)-[h(x)-(k h(x)+(1-k) h(b))]^{p} f(x) \\
&-\int_{x}^{b}\left([h(t)-(k h(x)+(1-k) h(b))]^{p}\right)^{\Delta} f(\sigma(t)) \Delta t \\
&=(1-k)^{p}[h(x)-h(a)]^{p} f(x)-k^{p}[h(a)-h(x)]^{p} f(a) \\
&+k^{p}[h(b)-h(x)]^{p} f(b)-(1-k)^{p}[h(x)-h(b)]^{p} f(x) \\
&-\left[\int_{a}^{x}\left([h(t)-((1-k) h(a)+k h(x))]^{p}\right)^{\Delta} f(\sigma(t)) \Delta t\right. \\
&\left.+\int_{x}^{b}\left([h(t)-(k h(x)+(1-k) h(b))]^{p}\right)^{\Delta} f(\sigma(t)) \Delta t\right] \\
&=(1-k)^{p} f(x)\left[[h(x)-h(a)]^{p}-[h(x)-h(b)]^{p}\right] \\
&+k^{p}\left[f(b)[h(b)-h(x)]^{p}-f(a)[h(a)-h(x)]^{p}\right] \\
&-\left[\int_{a}^{x}\left([h(t)-((1-k) h(a)+k h(x))]^{p}\right)^{\Delta} f(\sigma(t)) \Delta t\right. \\
&\left.+\int_{x}^{b}\left([h(t)-(k h(x)+(1-k) h(b))]^{p}\right)^{\Delta} f(\sigma(t)) \Delta t\right] \\
&=(1-k)^{p} f(x)\left[[h(x)-h(a)]^{p}+[h(b)-h(x)]^{p}\right] \\
&+k^{p}\left[f(b)[h(b)-h(x)]^{p}+f(a)[h(x)-h(a)]^{p}\right] \\
&-\left[\int_{a}^{x}\left([h(t)-((1-k) h(a)+k h(x))]^{p}\right)^{\Delta} f(\sigma(t)) \Delta t\right. \\
&+\int_{x}^{b}\left([h(t)-(k h(x)+(1-k) h(b))]^{p}\right)^{\Delta} f(\sigma(t)) \Delta t \\
& \\
&
\end{aligned}
$$

Applying the chain rule (2.2), we see that there exists $d \in[t, \sigma(t)]$ such that

$$
\left([h(t)-((1-k) h(a)+k h(x))]^{p}\right)^{\Delta}=p[h(d)-((1-k) h(a)+k h(x))]^{p-1} h^{\Delta}(t),
$$

and

$$
\left([h(t)-(k h(x)+(1-k) h(b))]^{p}\right)^{\Delta}=p[h(d)-(k h(x)+(1-k) h(b))]^{p-1} h^{\Delta}(t) .
$$

Since $h^{\Delta}(t) \geqslant 0$ and $\sigma(t) \geqslant d$, we have

$$
\left([h(t)-((1-k) h(a)+k h(x))]^{p}\right)^{\Delta} \leqslant p[h(\sigma(t))-((1-k) h(a)+k h(x))]^{p-1} w(t),
$$

and

$$
\left([h(t)-(k h(x)+(1-k) h(b))]^{p}\right)^{\Delta} \leqslant p[h(\sigma(t))-(k h(x)+(1-k) h(b))]^{p-1} w(t) .
$$


Applying the inequalities (3.1) and (3.2) on the term $[h(x)-h(a)]^{p}+[h(b)-h(x)]^{p}$, we get

$$
\begin{aligned}
{[h(x)-h(a)]^{p}+[h(b)-h(x)]^{p} } & \leqslant \beta[h(x)-h(a)+h(b)-h(x)]^{p} \\
& =\beta[h(b)-h(a)]^{p}=\beta\left(\int_{a}^{b} w(t) \Delta t\right)^{p} .
\end{aligned}
$$

Combining (3.6), (3.7), (3.8), and (3.9), we have that

$$
\begin{aligned}
& \int_{a}^{b} s^{p}(x, t) f^{\Delta}(t) \Delta t \\
& \leqslant \beta(1-k)^{p} f(x)\left(\int_{a}^{b} w(t) \Delta t\right)^{p}+k^{p}\left[f(a)\left(\int_{a}^{x} w(t) \Delta t\right)^{p}+f(b)\left(\int_{x}^{b} w(t) \Delta t\right)^{p}\right] \\
& \quad-p\left[\int_{a}^{x}[h(\sigma(t))-((1-k) h(a)+k h(x))]^{p-1} w(t) f(\sigma(t)) \Delta t\right. \\
& \left.\quad+\int_{x}^{b} p[h(\sigma(t))-(k h(x)+(1-k) h(b))]^{p-1} w(t) f(\sigma(t)) \Delta t\right] \\
& \leqslant \\
& \quad\left\{\beta(1-k)^{p} f(x)+k^{p}\left[\left(\frac{\int_{a}^{x} w(t) \Delta t}{\int_{a}^{b} w(t) \Delta t}\right)^{p} f(a)+\left(\frac{\int_{x}^{b} w(t) \Delta t}{\int_{a}^{b} w(t) \Delta t}\right)^{p} f(b)\right]\right\}\left(\int_{a}^{b} w(t) \Delta t\right)^{p} \\
& \quad-p\left[\int_{a}^{x}[h(\sigma(t))-((1-k) h(a)+k h(x))]^{p-1} w(t) f(\sigma(t)) \Delta t\right. \\
& \left.\quad+\int_{x}^{b} p[h(\sigma(t))-(k h(x)+(1-k) h(b))]^{p-1} w(t) f(\sigma(t)) \Delta t\right]
\end{aligned}
$$

which provides the desired inequality (3.5). The proof is complete.

Lemma 3.2. Let $\mathrm{a}, \mathrm{b} \in \mathbb{T}$ with $\mathrm{a}<\mathrm{b}, 0 \leqslant \mathrm{k} \leqslant 1, w:[\mathrm{a}, \mathrm{b}]_{\mathbb{T}} \rightarrow[0, \infty)$ be rd-continuous and positive function and $\mathrm{f}, \mathrm{h}:[\mathrm{a}, \mathrm{b}]_{\mathbb{T}} \rightarrow \mathbb{R}$ are $\Delta$-differentiable functions such that $\mathrm{h}^{\Delta}(\mathrm{t})=\mathrm{w}(\mathrm{t})$ on $[\mathrm{a}, \mathrm{b}]_{\mathbb{T}}$. Define

$$
s(x, t)= \begin{cases}h(t)-((1-k) h(a)+k h(x)), & a \leqslant t<x \\ h(t)-(k h(x)+(1-k) h(b)), & x \leqslant t \leqslant b\end{cases}
$$

Then for all $x \in[a, b]_{\mathbb{T}}$, we have

$$
\begin{aligned}
& \int_{a}^{b} s^{p}(x, t) f^{\Delta}(t) \Delta t \\
& \geqslant \\
& \quad\left\{\gamma(1-k)^{p} f(x)+k^{p}\left[\left(\frac{\int_{a}^{x} w(t) \Delta t}{\int_{a}^{b} w(t) \Delta t}\right)^{p} f(a)+\left(\frac{\int_{x}^{b} w(t) \Delta t}{\int_{a}^{b} w(t) \Delta t}\right)^{p} f(b)\right]\right\}\left(\int_{a}^{b} w(t) \Delta t\right)^{p} \\
& \quad-p\left[\int_{a}^{x}[h(t)-((1-k) h(a)+k h(x))]^{p-1} w(t) f(\sigma(t)) \Delta t\right. \\
& \left.\quad+\int_{x}^{b}[h(t)-(k h(x)+(1-k) h(b))]^{p-1} w(t) f(\sigma(t)) \Delta t\right]
\end{aligned}
$$

where

$$
\gamma= \begin{cases}1, & \text { if } 0 \leqslant p \leqslant 1 \\ 2^{1-p}, & \text { if } p<0, \text { or } p \geqslant 1\end{cases}
$$


Proof. By using integration by parts (2.3), we see that

$$
\begin{aligned}
& \int_{a}^{b} s^{p}(x, t) f^{\Delta}(t) \Delta t \\
&=\int_{a}^{x} s^{p}(x, t) f^{\Delta}(t) \Delta t+\int_{x}^{b} s^{p}(x, t) f^{\Delta}(t) \Delta t \\
&=\int_{a}^{x}[h(t)-((1-k) h(a)+k h(x))]^{p} f^{\Delta}(t) \Delta t+\int_{x}^{b}[h(t)-(k h(x)+(1-k) h(b))]^{p} f^{\Delta}(t) \Delta t \\
&=[h(x)-((1-k) h(a)+k h(x))]^{p} f(x)-[h(a)-((1-k) h(a)+k h(x))]^{p} f(a) \\
& \quad-\int_{a}^{x}\left([h(t)-((1-k) h(a)+k h(x))]^{p}\right)^{\Delta} f(\sigma(t)) \Delta t \\
&+[h(b)-(k h(x)+(1-k) h(b))]^{p} f(b)-[h(x)-(k h(x)+(1-k) h(b))]^{p} f(x) \\
& \quad-\int_{x}^{b}\left([h(t)-(k h(x)+(1-k) h(b))]^{p}\right)^{\Delta} f(\sigma(t)) \Delta t \\
&=(1-k)^{p}[h(x)-h(a)]^{p} f(x)-k^{p}[h(a)-h(x)]^{p} f(a) \\
&+k^{p}[h(b)-h(x)]^{p} f(b)-(1-k)^{p}[h(x)-h(b)]^{p} f(x) \\
& \quad-\left[\int_{a}^{x}\left([h(t)-((1-k) h(a)+k h(x))]^{p}\right)^{\Delta} f(\sigma(t)) \Delta t+\int_{x}^{b}\left([h(t)-(k h(x)+(1-k) h(b))]^{p}\right)^{\Delta} f(\sigma(t)) \Delta t\right] \\
&=(1-k)^{p} f(x)\left[[h(x)-h(a)]^{p}-[h(x)-h(b)]^{p}\right]+k^{p}\left[f(b)[h(b)-h(x)]^{p}-f(a)[h(a)-h(x)]^{p}\right] \\
&-\left[\int_{a}^{x}\left([h(t)-((1-k) h(a)+k h(x))]^{p}\right)^{\Delta} f(\sigma(t)) \Delta t+\int_{x}^{b}\left([h(t)-(k h(x)+(1-k) h(b))]^{p}\right)^{\Delta} f(\sigma(t)) \Delta t\right] \\
&=(1-k)^{p} f(x)\left[[h(x)-h(a)]^{p}+[h(b)-h(x)]^{p}\right]+k^{p}\left[f(b)[h(b)-h(x)]^{p}+f(a)[h(x)-h(a)]^{p}\right] \\
&-\left[\int_{a}^{x}\left([h(t)-((1-k) h(a)+k h(x))]^{p}\right)^{\Delta} f(\sigma(t)) \Delta t+\int_{x}^{b}\left([h(t)-(k h(x)+(1-k) h(b))]^{p}\right)^{\Delta} f(\sigma(t)) \Delta t\right] .
\end{aligned}
$$

Applying the chain rule (2.2), we see that there exists $d \in[t, \sigma(t)]$ such that

$$
\left([h(t)-((1-k) h(a)+k h(x))]^{p}\right)^{\Delta}=p[h(d)-((1-k) h(a)+k h(x))]^{p-1} h^{\Delta}(t),
$$

and

$$
\left([h(t)-(k h(x)+(1-k) h(b))]^{p}\right)^{\Delta}=p[h(d)-(k h(x)+(1-k) h(b))]^{p-1} h^{\Delta}(t) .
$$

Since $h^{\Delta}(t) \geqslant 0$ and $t \leqslant d$, we have

$$
\left([h(t)-((1-k) h(a)+k h(x))]^{p}\right)^{\Delta} \geqslant p[h(t)-((1-k) h(a)+k h(x))]^{p-1} w(t),
$$

and

$$
\left([h(t)-(k h(x)+(1-k) h(b))]^{p}\right)^{\Delta} \geqslant p[h(t)-(k h(x)+(1-k) h(b))]^{p-1} w(t) .
$$

Applying the inequalities (3.1), (3.2,) and (3.3) on the term $[h(x)-h(a)]^{p}+[h(b)-h(x)]^{p}$, we get

$$
\begin{aligned}
{[h(x)-h(a)]^{p}+[h(b)-h(x)]^{p} } & \geqslant \gamma[h(x)-h(a)+h(b)-h(x)]^{p} \\
& =\gamma[h(b)-h(a)]^{p}=\gamma\left(\int_{a}^{b} w(t) \Delta t\right)^{p} .
\end{aligned}
$$

Combining (3.11), (3.12), (3.13), and (3.14), we have that

$$
\int_{a}^{b} s^{p}(x, t) f^{\Delta}(t) \Delta t
$$




$$
\begin{aligned}
\geqslant & \gamma(1-k)^{p} f(x)\left(\int_{a}^{b} w(t) \Delta t\right)^{p}+k^{p}\left[f(a)\left(\int_{a}^{x} w(t) \Delta t\right)^{p}+f(b)\left(\int_{x}^{b} w(t) \Delta t\right)^{p}\right] \\
& -p\left[\int_{a}^{x}[h(t)-((1-k) h(a)+k h(x))]^{p-1} w(t) f(\sigma(t)) \Delta t\right. \\
& \left.+\int_{x}^{b} p[h(t)-(k h(x)+(1-k) h(b))]^{p-1} w(t) f(\sigma(t)) \Delta t\right] \\
\geqslant & \left\{\gamma(1-k)^{p} f(x)+k^{p}\left[\left(\frac{\int_{a}^{x} w(t) \Delta t}{\int_{a}^{b} w(t) \Delta t}\right)^{p} f(a)+\left(\frac{\int_{x}^{b} w(t) \Delta t}{\int_{a}^{b} w(t) \Delta t}\right)^{p} f(b)\right]\right\}\left(\int_{a}^{b} w(t) \Delta t\right)^{p} \\
& -p\left[\int_{a}^{x}[h(t)-((1-k) h(a)+k h(x))]^{p-1} w(t) f(\sigma(t)) \Delta t\right. \\
& \left.+\int_{x}^{b} p[h(t)-(k h(x)+(1-k) h(b))]^{p-1} w(t) f(\sigma(t)) \Delta t\right]
\end{aligned}
$$

which provides the desired inequality (3.10). The proof is complete.

In Lemmas 3.1 and 3.2, if we put $p=1$, we have the following weighted Montgomery identity on time scales proved in [30].

Corollary 3.3. Let $\mathrm{a}, \mathrm{b} \in \mathbb{T}$ with $\mathrm{a}<\mathrm{b}, 0 \leqslant \mathrm{k} \leqslant 1, w:[\mathrm{a}, \mathrm{b}]_{\mathbb{T}} \rightarrow[0, \infty)$ be rd-continuous and positive function and $\mathrm{f}, \mathrm{h}:[\mathrm{a}, \mathrm{b}]_{\mathbb{T}} \rightarrow \mathbb{R}$ are $\Delta$-differentiable functions such that $\mathrm{h}^{\Delta}(\mathrm{t})=w(\mathrm{t})$ on $[\mathrm{a}, \mathrm{b}]_{\mathbb{T}}$. Define

$$
s(x, t)= \begin{cases}h(t)-((1-k) h(a)+k h(x)), & a \leqslant t<x, \\ h(t)-(k h(x)+(1-k) h(b)), & x \leqslant t \leqslant b .\end{cases}
$$

Then

$$
\int_{a}^{b} s(x, t) f^{\Delta}(t) \Delta t=\left\{(1-k) f(x)+k\left[\frac{\int_{a}^{x} w(t) \Delta t}{\int_{a}^{b} w(t) \Delta t} f(a)+\frac{\int_{x}^{b} w(t) \Delta t}{\int_{a}^{b} w(t) \Delta t} f(b)\right]\right\} \int_{a}^{b} w(t) \Delta t-\int_{a}^{b} w(t) f(\sigma(t)) \Delta t .
$$

In Corollary 3.3, if $\mathbb{T}=\mathbb{R}$, then we have the following result proved in [27].

Corollary 3.4. Let $0<\mathrm{a}<\mathrm{b}, 0 \leqslant \mathrm{k} \leqslant 1$, and $\mathrm{f}, \mathrm{h}:[\mathrm{a}, \mathrm{b}]_{\mathbb{R}} \rightarrow \mathbb{R}$ are differentiable functions such that $\mathrm{h}^{\prime}(\mathrm{t})=\mathrm{w}(\mathrm{t})$ on $[\mathrm{a}, \mathrm{b}]_{\mathbb{R}}$. Define

$$
s(x, t)= \begin{cases}h(t)-((1-k) h(a)+k h(x)), & a \leqslant t<x \\ h(t)-(k h(x)+(1-k) h(b)), & x \leqslant t \leqslant b\end{cases}
$$

Then

$$
\int_{a}^{b} s(x, t) f^{\prime}(t) d t=\left\{(1-k) f(x)+k\left[\frac{\int_{a}^{x} w(t) d t}{\int_{a}^{b} w(t) d t} f(a)+\frac{\int_{x}^{b} w(t) d t}{\int_{a}^{b} w(t) d t} f(b)\right]\right\} \int_{a}^{b} w(t) d t-\int_{a}^{b} w(t) f(t) d t .
$$

In Corollary 3.3, if $\mathbb{T}=\mathbb{Z}$, then we have the following result proved in [30].

Corollary 3.5. Let $0<\mathrm{a}<\mathrm{b}, 0 \leqslant \mathrm{k} \leqslant 1$, and $\mathrm{f}, \mathrm{h}:[\mathrm{a}, \mathrm{b}]_{\mathbb{Z}} \rightarrow \mathbb{R}$, such that $\Delta \mathrm{h}(\mathrm{n})=w(\mathrm{n})$ on $[\mathrm{a}, \mathrm{b}]_{\mathbb{Z}}$. Define

$$
s(m, n)= \begin{cases}h(n)-((1-k) h(a)+k h(m)), & a \leqslant n<m-1, \\ h(n)-(k h(m)+(1-k) h(b)), & m \leqslant n \leqslant b-1 .\end{cases}
$$

Then

$$
\begin{aligned}
\sum_{n=a}^{b-1} s(m, n) \Delta f(n) \leqslant & \left\{(1-k) f(m)+k\left[\frac{\sum_{n=a}^{m-1} w(n)}{\sum_{n=a}^{b-1} w(n)} f(a)+\frac{\sum_{n=m}^{b-1} w(n)}{\sum_{n=a}^{b-1} w(n)} f(b)\right]\right\} \sum_{n=a}^{b-1} w(n) \\
& -\sum_{n=a}^{b-1} w(n) f(n+1) .
\end{aligned}
$$


In Corollary 3.3, if $\mathbb{T}=\mathrm{q}^{\mathbb{N}}$, then we have the following result proved in [30].

Corollary 3.6. Let $0<\mathrm{a}<\mathrm{b}, 0 \leqslant \mathrm{k} \leqslant 1, \mathrm{q}>1, \mathrm{a}=\mathrm{q}^{\mathrm{m}}$ and $\mathrm{b}=\mathrm{q}^{\mathrm{n}}$ with $\mathrm{m}<\mathrm{n}$ and $\mathrm{f}, \mathrm{h}:[\mathrm{a}, \mathrm{b}]_{\mathrm{q}^{\mathrm{N}}} \rightarrow \mathbb{R}$, such that $\Delta_{\mathrm{q}} \mathrm{h}\left(\mathrm{q}^{\mathrm{i}}\right)=w\left(\mathrm{q}^{\mathrm{i}}\right)$ on $[\mathrm{a}, \mathrm{b}]_{\mathrm{q}^{\mathbb{N}}}$. Define

$$
s\left(j, q^{i}\right)= \begin{cases}h\left(q^{i}\right)-\left((1-k) h\left(q^{m}\right)+k h(j)\right), & q^{m} \leqslant q^{i}<j-1, \\ h\left(q^{i}\right)-\left(k h(j)+(1-k) h\left(q^{n}\right)\right), & j \leqslant q^{i} \leqslant q^{n-1}\end{cases}
$$

Then

$$
\begin{aligned}
\sum_{i=m}^{n-1} s\left(j, q^{i}\right) \Delta_{q} f\left(q^{i}\right) \leqslant & \left\{(1-k) f(j)+k\left[\frac{\sum_{i=m}^{j-1} w\left(q^{i}\right)}{\sum_{i=m}^{n-1} w\left(q^{i}\right)} f\left(q^{m}\right)+\frac{\sum_{i=j}^{n-1} w\left(q^{i}\right)}{\sum_{i=m}^{n-1} w\left(q^{i}\right)} f\left(q^{n}\right)\right]\right\} \sum_{i=m}^{n-1} w\left(q^{i}\right) \\
& -\sum_{i=m}^{n-1} w\left(q^{i}\right) f\left(q^{i}\right) .
\end{aligned}
$$

In Corollary 3.3, if $h(t)=t$, then we have the following result proved in [46, Lemma 1].

Corollary 3.7. Let $\mathrm{a}, \mathrm{b} \in \mathbb{T}$ with $\mathrm{a}<\mathrm{b}, 0 \leqslant \mathrm{k} \leqslant 1$, and $\mathrm{f}:[\mathrm{a}, \mathrm{b}]_{\mathbb{T}} \rightarrow \mathbb{R}$ be $\Delta$-differentiable functions. Define

$$
s(x, t)= \begin{cases}t-((1-k) a+k x), & a \leqslant t<x \\ t-(k x+(1-k) b), & x \leqslant t \leqslant b\end{cases}
$$

Then

$$
(1-k) f(x)=\frac{1}{b-a} \int_{a}^{b} s(x, t) f^{\Delta}(t) \Delta t-k\left[\frac{x-a}{b-a} f(a)+\frac{b-x}{b-a} f(b)\right]+\frac{1}{b-a} \int_{a}^{b} f(\sigma(t)) \Delta t .
$$

In Corollary 3.7, if $\mathbb{T}=\mathbb{R}$, then we have the following result proved in [30].

Corollary 3.8. Let $0<\mathrm{a}<\mathrm{b}, 0 \leqslant \mathrm{k} \leqslant 1$, and $\mathrm{f}:[\mathrm{a}, \mathrm{b}]_{\mathbb{R}} \rightarrow \mathbb{R}$ be differentiable functions. Define

$$
s(x, t)= \begin{cases}t-((1-k) a+k x), & a \leqslant t<x \\ t-(k x+(1-k) b), & x \leqslant t \leqslant b\end{cases}
$$

Then

$$
(1-k) f(x)=\frac{1}{b-a} \int_{a}^{b} s(x, t) f^{\prime}(t) d t-k\left[\frac{x-a}{b-a} f(a)+\frac{b-x}{b-a} f(b)\right]+\frac{1}{b-a} \int_{a}^{b} f(t) d t .
$$

In Corollary 3.7, if $\mathbb{T}=\mathbb{Z}$, then we have the following result proved in [30].

Corollary 3.9. Let $0<\mathrm{a}<\mathrm{b}, 0 \leqslant \mathrm{k} \leqslant 1$, and $\mathrm{f}:[\mathrm{a}, \mathrm{b}]_{\mathbb{Z}} \rightarrow \mathbb{R}$. Define

$$
s(m, n)= \begin{cases}n-((1-k) a+k m), & a \leqslant n<m-1 \\ n-(k m+(1-k) b), & m \leqslant n \leqslant b-1\end{cases}
$$

Then

$$
(1-k) f(m)=\frac{1}{b-a} \sum_{n=a}^{b-1} s(m, n) \Delta f(n)-k\left[\frac{m-a}{b-a} f(a)+\frac{b-m}{b-a} f(b)\right]+\frac{1}{b-a} \sum_{n=a}^{b-1} f(n+1) .
$$
3.1].

In Corollary 3.7, if $k=0$, then we have the Montgomery identity on time scales proved in [11, Lemma

Corollary 3.10. Let $\mathrm{a}, \mathrm{b} \in \mathbb{T}$ with $\mathrm{a}<\mathrm{b}$, and $\mathrm{f}:[\mathrm{a}, \mathrm{b}]_{\mathbb{T}} \rightarrow \mathbb{R}$ be $\Delta$-differentiable functions. Define

$$
s(x, t)= \begin{cases}t-a, & a \leqslant t<x \\ t-b, & x \leqslant t \leqslant b\end{cases}
$$

Then

$$
f(x)=\frac{1}{b-a} \int_{a}^{b} s(x, t) f^{\Delta}(t) \Delta t+\frac{1}{b-a} \int_{a}^{b} f^{\sigma}(t) \Delta t
$$




\section{Main results}

In this section, by using weighted Montgomery inequality and it reversed on time scales proved in the previous section, we prove some weighted Ostrowski and Trapezoid inequalities on time scales.

4.1. Weighted Ostrowski's type inequalities

Theorem 4.1. Let $\mathrm{a}, \mathrm{b} \in \mathbb{T}$ with $\mathrm{a}<\mathrm{b}, 0 \leqslant \mathrm{k} \leqslant 1, \mathrm{M}=\sup _{\mathrm{a}<\mathrm{t}<\mathrm{b}}\left|\mathrm{f}^{\Delta}(\mathrm{t})\right|<\infty, w:[\mathrm{a}, \mathrm{b}]_{\mathbb{T}} \rightarrow[0, \infty)$ be rdcontinuous and positive function and $\mathrm{f}, \mathrm{h}:[\mathrm{a}, \mathrm{b}]_{\mathbb{T}} \rightarrow \mathbb{R}$ are $\Delta$-differentiable functions such that $\mathrm{h}^{\Delta}(\mathrm{t})=w(\mathrm{t})$ on $[a, b]_{\mathbb{T}}$. Then for all $x \in[a, b]_{\mathbb{T}}$, we have

$$
\begin{aligned}
& \mid\left\{\gamma(1-k)^{p} f(x)+k^{p}\left[\left(\frac{\int_{a}^{x} w(t) \Delta t}{\int_{a}^{b} w(t) \Delta t}\right)^{p} f(a)+\left(\frac{\int_{x}^{b} w(t) \Delta t}{\int_{a}^{b} w(t) \Delta t}\right)^{p} f(b)\right]\right\}\left(\int_{a}^{b} w(t) \Delta t\right)^{p} \\
& \quad-p\left[\int_{a}^{x}[h(t)-((1-k) h(a)+k h(x))]^{p-1} w(t) f(\sigma(t)) \Delta t\right. \\
& \left.\quad+\int_{x}^{b}[h(t)-(k h(x)+(1-k) h(b))]^{p-1} w(t) f(\sigma(t)) \Delta t\right]\left|\leqslant M \int_{a}^{b}\right| s^{p}(x, t) \mid \Delta t
\end{aligned}
$$

where

$$
\gamma= \begin{cases}1, & \text { if } 0 \leqslant p \leqslant 1 \\ 2^{1-p}, & \text { if } p<0, \text { or } p \geqslant 1\end{cases}
$$

Proof. From Lemma 3.2, we see that

$$
\begin{aligned}
& \left\{\gamma(1-k)^{p} f(x)+k^{p}\left[\left(\frac{\int_{a}^{x} w(t) \Delta t}{\int_{a}^{b} w(t) \Delta t}\right)^{p} f(a)+\left(\frac{\int_{x}^{b} w(t) \Delta t}{\int_{a}^{b} w(t) \Delta t}\right)^{p} f(b)\right]\right\}\left(\int_{a}^{b} w(t) \Delta t\right)^{p} \\
& -p\left[\int_{a}^{x}[h(t)-((1-k) h(a)+k h(x))]^{p-1} w(t) f(\sigma(t)) \Delta t\right. \\
& \left.+\int_{x}^{b}[h(t)-(k h(x)+(1-k) h(b))]^{p-1} w(t) f(\sigma(t)) \Delta t\right] \leqslant \int_{a}^{b} s^{p}(x, t) f^{\Delta}(t) \Delta t .
\end{aligned}
$$

Taking the absolute value, we have

$$
\begin{aligned}
& \mid\left\{\gamma(1-k)^{p} f(x)+k^{p}\left[\left(\frac{\int_{a}^{x} w(t) \Delta t}{\int_{a}^{b} w(t) \Delta t}\right)^{p} f(a)+\left(\frac{\int_{x}^{b} w(t) \Delta t}{\int_{a}^{b} w(t) \Delta t}\right)^{p} f(b)\right]\right\}\left(\int_{a}^{b} w(t) \Delta t\right)^{p} \\
& \quad-p\left[\int_{a}^{x}[h(t)-((1-k) h(a)+k h(x))]^{p-1} w(t) f(\sigma(t)) \Delta t\right. \\
& \left.\quad+\int_{x}^{b}[h(t)-(k h(x)+(1-k) h(b))]^{p-1} w(t) f(\sigma(t)) \Delta t\right]\left|\leqslant \int_{a}^{b}\right| s^{p}(x, t)|| f^{\Delta}(t)\left|\Delta t \leqslant M \int_{a}^{b}\right| s^{p}(x, t) \mid \Delta t,
\end{aligned}
$$

which provides the desired inequality (4.1). The proof is complete.

In Theorem 4.1, if we put $p=1$, we have the following weighted Ostrowski type inequality on time scales proved in [30, Theorem 6].

Corollary 4.2. Let $\mathrm{a}, \mathrm{b} \in \mathbb{T}$ with $\mathrm{a}<\mathrm{b}, 0 \leqslant \mathrm{k} \leqslant 1, \mathrm{M}=\sup _{\mathrm{a}<\mathrm{t}<\mathrm{b}}\left|\mathrm{f}^{\Delta}(\mathrm{t})\right|<\infty, w:[\mathrm{a}, \mathrm{b}]_{\mathbb{T}} \rightarrow[0, \infty)$ be $r d$-continuous and positive function and $\mathrm{f}, \mathrm{h}:[\mathrm{a}, \mathrm{b}]_{\mathbb{T}} \rightarrow \mathbb{R}$ are $\Delta$-differentiable functions such that $\mathrm{h}^{\Delta}(\mathrm{t})=w(\mathrm{t})$ on $[\mathrm{a}, \mathrm{b}]_{\mathbb{T}}$. Define

$$
s(x, t)= \begin{cases}h(t)-((1-k) h(a)+k h(x)), & a \leqslant t<x \\ h(t)-(k h(x)+(1-k) h(b)), & x \leqslant t \leqslant b\end{cases}
$$


Then

$$
\begin{aligned}
& \mid\left\{(1-k) f(x)+k\left[\frac{\int_{a}^{x} w(t) \Delta t}{\int_{a}^{b} w(t) \Delta t} f(a)+\frac{\int_{x}^{b} w(t) \Delta t}{\int_{a}^{b} w(t) \Delta t} f(b)\right]\right\} \int_{a}^{b} w(t) \Delta t \\
& -\int_{a}^{b} w(t) f(\sigma(t)) \Delta t\left|\leqslant M \int_{a}^{b}\right|(s(x, t)) \mid \Delta t .
\end{aligned}
$$

In Corollary 4.2, if $\mathbb{T}=\mathbb{R}$, then we have the following result proved in [30].

Corollary 4.3. Let $0<\mathrm{a}<\mathrm{b}, 0 \leqslant \mathrm{k} \leqslant 1, \mathrm{M}=\sup _{\mathrm{a}<\mathrm{t}<\mathrm{b}}\left|\mathrm{f}^{\prime}(\mathrm{t})\right|<\infty$ and $\mathrm{f}, \mathrm{h}:[\mathrm{a}, \mathrm{b}]_{\mathbb{R}} \rightarrow \mathbb{R}$ are differentiable functions such that $\mathrm{h}^{\prime}(\mathrm{t})=w(\mathrm{t})$ on $[\mathrm{a}, \mathrm{b}]_{\mathbb{R}}$. Define

$$
s(x, t)= \begin{cases}h(t)-((1-k) h(a)+k h(x)), & a \leqslant t<x \\ h(t)-(k h(x)+(1-k) h(b)), & x \leqslant t \leqslant b\end{cases}
$$

Then

$$
\begin{aligned}
& \mid\left\{(1-k) f(x)+k\left[\frac{\int_{a}^{x} w(t) d t}{\int_{a}^{b} w(t) d t} f(a)+\frac{\int_{x}^{b} w(t) d t}{\int_{a}^{b} w(t) d t} f(b)\right]\right\} \int_{a}^{b} w(t) d t \\
& \quad-\int_{a}^{b} w(t) f(t) d t\left|\leqslant M \int_{a}^{b}\right|(s(x, t)) \mid d t .
\end{aligned}
$$

In Corollary 4.2, if $\mathbb{T}=\mathbb{Z}$, then we have the following result proved in [30].

Corollary 4.4. Let $0<\mathrm{a}<\mathrm{b}, 0 \leqslant \mathrm{k} \leqslant 1, \mathrm{M}=\sup _{\mathrm{a}<\mathrm{t}<\mathrm{b}-1}|\Delta \mathrm{f}(\mathrm{t})|<\infty$ and $\mathrm{f}, \mathrm{h}:[\mathrm{a}, \mathrm{b}]_{\mathbb{Z}} \rightarrow \mathbb{R}$, such that $\Delta \mathrm{h}(\mathrm{n})=w(\mathrm{n})$ on $[\mathrm{a}, \mathrm{b}]_{\mathbb{Z}}$. Define

$$
s(m, n)= \begin{cases}h(n)-((1-k) h(a)+k h(m)), & a \leqslant n<m-1 \\ h(n)-(k h(m)+(1-k) h(b)), & m \leqslant n \leqslant b-1\end{cases}
$$

Then

$$
\begin{aligned}
& \mid\left\{(1-k) f(m)+k\left[\frac{\sum_{n=a}^{m-1} w(n)}{\sum_{n=a}^{b-1} w(n)} f(a)+\frac{\sum_{n=m}^{b-1} w(n)}{\sum_{n=a}^{b-1} w(n)} f(b)\right]\right\} \sum_{n=a}^{b-1} w(n) \\
& \quad-\sum_{n=a}^{b-1} w(n) f(n+1)\left|\leqslant M \sum_{n=a}^{b-1}\right|(s(m, n)) \mid .
\end{aligned}
$$

In Corollary 4.2, if $\mathbb{T}=q^{\mathbb{N}}$, then we have the following result proved in [30].

Corollary 4.5. Let $0<\mathrm{a}<\mathrm{b}, 0 \leqslant \mathrm{k} \leqslant 1, \mathrm{M}=\sup _{\mathrm{q}^{\mathrm{m}}<\mathrm{t}<\mathrm{q}^{\mathrm{n}}}\left|\Delta_{\mathrm{q}} \mathrm{f}(\mathrm{t})\right|<\infty, \mathrm{q}>1, \mathrm{a}=\mathrm{q}^{\mathrm{m}}$ and $\mathrm{b}=\mathrm{q}^{\mathrm{n}}$ with $\mathrm{m}<\mathrm{n}$ and $\mathrm{f}, \mathrm{h}:[\mathrm{a}, \mathrm{b}]_{\mathrm{q}^{\mathbb{N}}} \rightarrow \mathbb{R}$, such that $\Delta_{\mathrm{q}} \mathrm{h}\left(\mathrm{q}^{\mathrm{i}}\right)=\mathcal{w}\left(\mathrm{q}^{\mathrm{i}}\right)$ on $[\mathrm{a}, \mathrm{b}]_{\mathrm{q}^{\mathbb{N}}}$. Define

$$
s\left(j, q^{i}\right)= \begin{cases}h\left(q^{i}\right)-\left((1-k) h\left(q^{m}\right)+k h(j)\right), & q^{m} \leqslant q^{i}<j-1, \\ h\left(q^{i}\right)-\left(k h(j)+(1-k) h\left(q^{n}\right)\right), & j \leqslant q^{i} \leqslant q^{n-1}\end{cases}
$$

Then

$$
\begin{aligned}
& \mid\left\{(1-k) f(j)+k\left[\frac{\sum_{i=m}^{j-1} w\left(q^{i}\right)}{\sum_{i=m}^{n-1} w\left(q^{i}\right)} f\left(q^{m}\right)+\frac{\sum_{i=j}^{n-1} w\left(q^{i}\right)}{\sum_{i=m}^{n-1} w\left(q^{i}\right)} f\left(q^{n}\right)\right]\right\} \sum_{i=m}^{n-1} w\left(q^{i}\right) \\
& \quad-\sum_{i=m}^{n-1} w\left(q^{i}\right) f\left(q^{i}\right)\left|\leqslant M \sum_{i=m}^{n-1}\right|\left(s\left(j, q^{i}\right)\right) \mid .
\end{aligned}
$$


In Corollary 4.2, if $h(t)=t$, then we have the following result proved in [30].

Corollary 4.6. Let $\mathrm{a}, \mathrm{b} \in \mathbb{T}$ with $\mathrm{a}<\mathrm{b}, 0 \leqslant \mathrm{k} \leqslant 1, \mathrm{M}=\sup _{\mathrm{a}<\mathrm{t}<\mathrm{b}}\left|\mathrm{f}^{\Delta}(\mathrm{t})\right|<\infty$ and $\mathrm{f}:[\mathrm{a}, \mathrm{b}]_{\mathbb{T}} \rightarrow \mathbb{R}$ be $\Delta$-differentiable functions. Define

$$
s(x, t)= \begin{cases}t-((1-k) a+k x), & a \leqslant t<x \\ t-(k x+(1-k) b), & x \leqslant t \leqslant b\end{cases}
$$

Then

$$
\begin{aligned}
& \left|(1-k) f(x)+k\left[\frac{x-a}{b-a} f(a)+\frac{b-x}{b-a} f(b)\right]-\frac{1}{b-a} \int_{a}^{b} f(\sigma(t)) \Delta t\right| \\
& \leqslant \frac{M}{b-a}\left[h_{2}((1-k) a+k t, a)+h_{2}(t,(1-k) a+k t)\right. \\
& \left.\quad+h_{2}(t, k t+(1-k) b)+h_{2}(b, k t+(1-k) b)\right] .
\end{aligned}
$$

In Corollary 4.6, if $\mathbb{T}=\mathbb{R}$, then we have the following result proved in [30].

Corollary 4.7. Let $0<\mathrm{a}<\mathrm{b}, 0 \leqslant \mathrm{k} \leqslant 1, \mathrm{M}=\sup _{\mathrm{a}<\mathrm{t}<\mathrm{b}}\left|\mathrm{f}^{\prime}(\mathrm{t})\right|<\infty$ and $\mathrm{f}:[\mathrm{a}, \mathrm{b}]_{\mathbb{R}} \rightarrow \mathbb{R}$ be differentiable functions. Then

$$
\left|(1-k) f(x)+k\left[\frac{x-a}{b-a} f(a)+\frac{b-x}{b-a} f(b)\right]-\frac{1}{b-a} \int_{a}^{b} f(t) d t\right| \leqslant \frac{M\left(2 k^{2}-2 k+1\right)}{2}\left[\frac{(a-t)^{2}+(b-t)^{2}}{b-a}\right] .
$$

In Corollary 4.6, if $\mathbb{T}=\mathbb{Z}$, then we have the following result proved in [30].

Corollary 4.8. Let $0<\mathrm{a}<\mathrm{b}, 0 \leqslant \mathrm{k} \leqslant 1, \mathrm{M}=\sup _{\mathrm{a}<\mathrm{n}<\mathrm{b}-1}|\Delta \mathrm{f}(\mathrm{n})|<\infty$ and $\mathrm{f}:[\mathrm{a}, \mathrm{b}]_{\mathbb{Z}} \rightarrow \mathbb{R}$. Then

$$
\begin{aligned}
& \left|(1-k) f(m)+k\left[\frac{m-a}{b-a} f(a)+\frac{b-m}{b-a} f(b)\right]-\frac{1}{b-a} \sum_{n=a}^{b-1} f(n+1)\right| \\
& \leqslant \frac{M}{b-a}\left[\left(2 k^{2}-2 k+1\right)\left(t-\frac{a+b}{2}\right)^{2}+(2 k-1)\left(t-\frac{a+b}{2}\right)+\left(\frac{b-a}{2}\right)^{2}\right],
\end{aligned}
$$

In Corollary 4.6, if $k=0$, then we have the Ostrowski inequality on time scales proved in [11, Theorem 3.5].

Corollary 4.9. Let $\mathrm{a}, \mathrm{b} \in \mathbb{T}$ with $\mathrm{a}<\mathrm{b}, \mathrm{M}=\sup _{\mathrm{a}<\mathrm{t}<\mathrm{b}}\left|\mathrm{f}^{\Delta}(\mathrm{t})\right|<\infty$ and $\mathrm{f}:[\mathrm{a}, \mathrm{b}]_{\mathbb{T}} \rightarrow \mathbb{R}$ be $\Delta$-differentiable functions. Then

$$
\left|f(x)-\frac{1}{b-a} \int_{a}^{b} f(\sigma(t)) \Delta t\right| \leqslant \frac{M}{b-a}\left[h_{2}(t, a)+h_{2}(t, b)\right] .
$$

\subsection{Weighted Trapezoid's type inequalities}

Theorem 4.10. Let $\mathrm{a}, \mathrm{b} \in \mathbb{T}$ with $\mathrm{a}<\mathrm{b}, 0 \leqslant \mathrm{k} \leqslant 1, \mathrm{M}=\sup _{\mathrm{a}<\mathrm{t}<\mathrm{b}}\left|\mathrm{f}^{\Delta}(\mathrm{t})\right|<\infty, \mathrm{N}=\sup _{\mathrm{a}<\mathrm{t}<\mathrm{b}}\left|\mathrm{f}^{\Delta}(\sigma(\mathrm{t}))\right|<\infty$, $w:[a, b]_{\mathbb{T}} \rightarrow[0, \infty)$ be rd-continuous and positive function and $\mathrm{f}, \mathrm{h}:[\mathrm{a}, \mathrm{b}]_{\mathbb{T}} \rightarrow \mathbb{R}$ are $\Delta$-differentiable functions 
such that $\mathrm{h}^{\Delta}(\mathrm{t})=w(\mathrm{t})$ on $[\mathrm{a}, \mathrm{b}]_{\mathbb{T}}$. Then for all $\mathrm{x} \in[\mathrm{a}, \mathrm{b}]_{\mathbb{T}}$, we have

$$
\begin{aligned}
& \mid \gamma(1-k)^{p}\left(f^{2}(b)-f^{2}(a)\right) \\
& \quad+k^{p}\left[-\frac{f(a)+f(\sigma(a))}{\left(\int_{a}^{b} w(t) \Delta t\right)^{p}} \int_{a}^{b} p\left(\int_{a}^{x} w(t) \Delta t\right)^{p-1} w(x) f(\sigma(x) \Delta x\right. \\
& \quad+\frac{f(b)+f(\sigma(b))}{\left(\int_{a}^{b} w(t) \Delta t\right)^{p}} \int_{a}^{b} p\left(\int_{x}^{b} w(t) \Delta t\right)^{p-1} w(x) f(\sigma(x) \Delta x]+k^{p}(f(b) f(\sigma(a))-f(a) f(\sigma(b)) \\
& \quad-p \frac{f(b)-f(a)}{\left(\int_{a}^{b} w(t) \Delta t\right)^{p}}\left[\int_{a}^{x}[h(t)-((1-k) h(a)+k h(x))]^{p-1} w(t)\left(f(\sigma(t))+f\left(\sigma^{2}(t)\right)\right) \Delta t\right. \\
& \left.\quad+\int_{x}^{b}[h(t)-(k h(x)+(1-k) h(b))]^{p-1} w(t)\left(f(\sigma(t))+f\left(\sigma^{2}(t)\right)\right) \Delta t\right] \mid \\
& \leqslant \frac{M(M+N)}{\left(\int_{a}^{b} w(t) \Delta t\right)^{p}} \int_{a}^{b}\left(\int_{a}^{b}\left|s^{p}(x, t)\right| \Delta t\right) \Delta x,
\end{aligned}
$$

where

$$
\gamma= \begin{cases}1, & \text { if } 0 \leqslant p \leqslant 1 \\ 2^{1-p}, & \text { if } p<0, \text { or } p \geqslant 1\end{cases}
$$

Proof. From Lemma 3.2 we see that

$$
\begin{aligned}
\gamma(1-k)^{p} f(x) \leqslant & -k^{p}\left[\left(\frac{\int_{a}^{x} w(t) \Delta t}{\int_{a}^{b} w(t) \Delta t}\right)^{p} f(a)+\left(\frac{\int_{x}^{b} w(t) \Delta t}{\int_{a}^{b} w(t) \Delta t}\right)^{p} f(b)\right] \\
& +\frac{p}{\left(\int_{a}^{b} w(t) \Delta t\right)^{p}}\left[\int_{a}^{x}[h(t)-((1-k) h(a)+k h(x))]^{p-1} w(t) f(\sigma(t)) \Delta t\right. \\
& \left.+\int_{x}^{b}[h(t)-(k h(x)+(1-k) h(b))]^{p-1} w(t) f(\sigma(t)) \Delta t\right] \\
& +\frac{1}{\left(\int_{a}^{b} w(t) \Delta t\right)^{p}} \int_{a}^{b} s^{p}(x, t) f^{\Delta}(t) \Delta t
\end{aligned}
$$

and

$$
\begin{aligned}
\gamma(1-k)^{p} f(\sigma(x)) \leqslant & -k^{p}\left[\left(\frac{\int_{a}^{x} w(t) \Delta t}{\int_{a}^{b} w(t) \Delta t}\right)^{p} f(\sigma(a))+\left(\frac{\int_{x}^{b} w(t) \Delta t}{\int_{a}^{b} w(t) \Delta t}\right)^{p} f(\sigma(b))\right] \\
& +\frac{p}{\left(\int_{a}^{b} w(t) \Delta t\right)^{p}}\left[\int_{a}^{x}[h(t)-((1-k) h(a)+k h(x))]^{p-1} w(t) f\left(\sigma^{2}(t)\right) \Delta t\right. \\
& \left.+\int_{x}^{b}[h(t)-(k h(x)+(1-k) h(b))]^{p-1} w(t) f\left(\sigma^{2}(t)\right) \Delta t\right] \\
& +\frac{1}{\left(\int_{a}^{b} w(t) \Delta t\right)^{p}} \int_{a}^{b} s^{p}(x, t) f^{\Delta}(\sigma(t)) \Delta t .
\end{aligned}
$$


Combining (4.3) and (4.4), we get

$$
\begin{aligned}
& \gamma(1-k)^{p}(f(x)+f(\sigma(x))) \\
& \leqslant-k^{p}\left[\left(\frac{\int_{a}^{x} w(t) \Delta t}{\int_{a}^{b} w(t) \Delta t}\right)^{p}(f(a)+f(\sigma(a)))+\left(\frac{\int_{x}^{b} w(t) \Delta t}{\int_{a}^{b} w(t) \Delta t}\right)^{p}(f(b)+f(\sigma(b)))\right] \\
&+\frac{p}{\left(\int_{a}^{b} w(t) \Delta t\right)^{p}}\left[\int_{a}^{x}[h(t)-((1-k) h(a)+k h(x))]^{p-1} w(t)\left(f(\sigma(t))+f\left(\sigma^{2}(t)\right)\right) \Delta t\right. \\
&\left.+\int_{x}^{b}[h(t)-(k h(x)+(1-k) h(b))]^{p-1} w(t)\left(f(\sigma(t))+f\left(\sigma^{2}(t)\right)\right) \Delta t\right] \\
&+\frac{1}{\left(\int_{a}^{b} w(t) \Delta t\right)^{p}} \int_{a}^{b} s^{p}(x, t)\left(f^{\Delta}(t)+f^{\Delta}(\sigma(t))\right) \Delta t .
\end{aligned}
$$

Multiplying (4.5) by $f^{\Delta}(x)$, and the relation (2.1) and integrating function at $[a, b]$, we have

$$
\begin{aligned}
& \gamma(1-k)^{p}\left(f^{2}(b)-f^{2}(a)\right) \\
& \leqslant- k^{p}\left[\frac{(f(a)+f(\sigma(a)))}{\left(\int_{a}^{b} w(t) \Delta t\right)^{p}} \int_{a}^{b} f^{\Delta}(x)\left(\int_{a}^{x} w(t) \Delta t\right)^{p} \Delta x\right. \\
&\left.+\frac{(f(b)+f(\sigma(b)))}{\left(\int_{a}^{b} w(t) \Delta t\right)^{p}} \int_{a}^{b} f^{\Delta}(x)\left(\int_{x}^{b} w(t) \Delta t\right)^{p} \Delta x\right] \\
&+\frac{p(f(b)-f(a))}{\left(\int_{a}^{b} w(t) \Delta t\right)^{p}}\left[\int_{a}^{x}[h(t)-((1-k) h(a)+k h(x))]^{p-1} w(t)\left(f(\sigma(t))+f\left(\sigma^{2}(t)\right)\right) \Delta t\right. \\
&\left.+\int_{x}^{b}[h(t)-(k h(x)+(1-k) h(b))]^{p-1} w(t)\left(f(\sigma(t))+f\left(\sigma^{2}(t)\right)\right) \Delta t\right] \\
&+\frac{1}{\left(\int_{a}^{b} w(t) \Delta t\right)^{p}} \int_{a}^{b} f^{\Delta}(x)\left(\int_{a}^{b}(s(x, t))^{p}\left(f^{\Delta}(t)+f^{\Delta}(\sigma(t))\right) \Delta t\right) \Delta x .
\end{aligned}
$$

From (4.6) and using the relations

$$
\int_{a}^{b} f^{\Delta}(x)\left(\int_{a}^{x} w(t) \Delta t\right)^{p} \Delta x=f(b)\left(\int_{a}^{b} w(t) \Delta t\right)^{p}-\int_{a}^{b} p\left(\int_{a}^{x} w(t) \Delta t\right)^{p-1} w(x) f(\sigma(x) \Delta x
$$

and

$$
\int_{a}^{b} f^{\Delta}(x)\left(\int_{x}^{b} w(t) \Delta t\right)^{p} \Delta x=-f(a)\left(\int_{a}^{b} w(t) \Delta t\right)^{p}+\int_{a}^{b} p\left(\int_{x}^{b} w(t) \Delta t\right)^{p-1} w(x) f(\sigma(x) \Delta x,
$$

we obtain

$$
\begin{aligned}
& \gamma(1-k)^{p}\left(f^{2}(b)-f^{2}(a)\right) \\
& +k^{p}\left[-\frac{f(a)+f(\sigma(a))}{\left(\int_{a}^{b} w(t) \Delta t\right)^{p}} \int_{a}^{b} p\left(\int_{a}^{x} w(t) \Delta t\right)^{p-1} w(x) f(\sigma(x) \Delta x\right.
\end{aligned}
$$




$$
\begin{aligned}
& +\frac{f(b)+f(\sigma(b))}{\left(\int_{a}^{b} w(t) \Delta t\right)^{p}} \int_{a}^{b} p\left(\int_{x}^{b} w(t) \Delta t\right)^{p-1} w(x) f(\sigma(x) \Delta x]+k^{p}(f(b) f(\sigma(a))-f(a) f(\sigma(b)) \\
& -p \frac{f(b)-f(a)}{\left(\int_{a}^{b} w(t) \Delta t\right)^{p}}\left[\int_{a}^{x}[h(t)-((1-k) h(a)+k h(x))]^{p-1} w(t)\left(f(\sigma(t))+f\left(\sigma^{2}(t)\right)\right) \Delta t\right. \\
& \left.+\int_{x}^{b}[h(t)-(k h(x)+(1-k) h(b))]^{p-1} w(t)\left(f(\sigma(t))+f\left(\sigma^{2}(t)\right)\right) \Delta t\right] \\
& \leqslant \frac{1}{\left(\int_{a}^{b} w(t) \Delta t\right)^{p}} \int_{a}^{b} f^{\Delta}(x)\left(\int_{a}^{b} s^{p}(x, t)\left(f^{\Delta}(t)+f^{\Delta}(\sigma(t))\right) \Delta t\right) \Delta x .
\end{aligned}
$$

Taking the absolute value we have

$$
\begin{aligned}
& \mid \gamma(1-k)^{p}\left(f^{2}(b)-f^{2}(a)\right) \\
& \quad+k^{p}\left[-\frac{f(a)+f(\sigma(a))}{\left(\int_{a}^{b} w(t) \Delta t\right)^{p}} \int_{a}^{b} p\left(\int_{a}^{x} w(t) \Delta t\right)^{p-1} w(x) f(\sigma(x) \Delta x\right. \\
& \quad+\frac{f(b)+f(\sigma(b))}{\left(\int_{a}^{b} w(t) \Delta t\right)^{p}} \int_{a}^{b} p\left(\int_{x}^{b} w(t) \Delta t\right)^{p-1} w(x) f(\sigma(x) \Delta x]+k^{p}(f(b) f(\sigma(a))-f(a) f(\sigma(b)) \\
& \quad-p \frac{f(b)-f(a)}{\left(\int_{a}^{b} w(t) \Delta t\right)^{p}}\left[\int_{a}^{x}[h(t)-((1-k) h(a)+k h(x))]^{p-1} w(t)\left(f(\sigma(t))+f\left(\sigma^{2}(t)\right)\right) \Delta t\right. \\
& \left.\quad+\int_{x}^{b}[h(t)-(k h(x)+(1-k) h(b))]^{p-1} w(t)\left(f(\sigma(t))+f\left(\sigma^{2}(t)\right)\right) \Delta t\right] \mid \\
& \leqslant\left|\frac{1}{\left(\int_{a}^{b} w(t) \Delta t\right)^{p}} \int_{a}^{b} f^{\Delta}(x)\left(\int_{a}^{b} s^{p}(x, t)\left(f^{\Delta}(t)+f^{\Delta}(\sigma(t))\right) \Delta t\right) \Delta x\right| \\
& \leqslant
\end{aligned}
$$

which provides the desired inequality (4.2). The proof is complete.

In Theorem 4.10 , if we put $p=1$, we have the following weighted Trapezoid type inequality on time scales proved in [30, Theorem 7].

Corollary 4.11. Let $\mathrm{a}, \mathrm{b} \in \mathbb{T}$ with $\mathrm{a}<\mathrm{b}, 0 \leqslant \mathrm{k} \leqslant 1, \mathrm{M}=\sup _{\mathrm{a}<\mathrm{t}<\mathrm{b}}\left|\mathrm{f}^{\Delta}(\mathrm{t})\right|<\infty, \mathrm{N}=\sup _{\mathrm{a}<\mathrm{t}<\mathrm{b}}\left|\mathrm{f}^{\Delta}(\sigma(\mathrm{t}))\right|<\infty$, $w:[a, b]_{\mathbb{T}} \rightarrow[0, \infty)$ be rd-continuous and positive functions and $f, h:[a, b]_{\mathbb{T}} \rightarrow \mathbb{R}$ are $\Delta$-differentiable functions such that $\mathrm{h}^{\Delta}(\mathrm{t})=\mathrm{w}(\mathrm{t})$ on $[\mathrm{a}, \mathrm{b}]_{\mathbb{T}}$. Define

$$
s(x, t)= \begin{cases}h(t)-((1-k) h(a)+k h(x)), & a \leqslant t<x \\ h(t)-(k h(x)+(1-k) h(b)), & x \leqslant t \leqslant b\end{cases}
$$

Then

$$
\mid(1-k)\left(f^{2}(b)-f^{2}(a)\right)+\left[(k-1) \frac{f(b)-f(a)}{\int_{a}^{b} w(t) \Delta t}+k \frac{f(\sigma(b))-f(\sigma(a))}{\int_{a}^{b} w(t) \Delta t}\right] \int_{a}^{b} w(x) f(\sigma(x) \Delta x
$$




$$
\begin{aligned}
& \quad+k(f(\sigma(a)) f(b)-f(\sigma(b)) f(a))-\frac{f(b)-f(a)}{\int_{a}^{b} w(t) \Delta t} \int_{a}^{b} w(t) f\left(\sigma^{2}(t)\right) \Delta t \mid \\
& \leqslant \frac{M(M+N)}{\int_{a}^{b} w(t) \Delta t} \int_{a}^{b}\left(\int_{a}^{b}|(s(x, t))| \Delta t\right) \Delta x .
\end{aligned}
$$

In Corollary 4.11, if $\mathbb{T}=\mathbb{R}$, then we have the following result proved in [30].

Corollary 4.12. Let $0<\mathrm{a}<\mathrm{b}, 0 \leqslant \mathrm{k} \leqslant 1, \mathrm{M}=\sup _{\mathrm{a}<\mathrm{t}<\mathrm{b}}\left|\mathrm{f}^{\prime}(\mathrm{t})\right|<\infty$ and $\mathrm{f}, \mathrm{h}:[\mathrm{a}, \mathrm{b}]_{\mathbb{R}} \rightarrow \mathbb{R}$ are differentiable functions such that $\mathrm{h}^{\prime}(\mathrm{t})=w(\mathrm{t})$ on $[\mathrm{a}, \mathrm{b}]_{\mathbb{R}}$. Define

$$
s(x, t)= \begin{cases}h(t)-((1-k) h(a)+k h(x)), & a \leqslant t<x \\ h(t)-(k h(x)+(1-k) h(b)), & x \leqslant t \leqslant b\end{cases}
$$

Then

$$
\left|(1-k) \frac{f^{2}(b)-f^{2}(a)}{2}+(k-1) \frac{f(b)-f(a)}{2 \int_{a}^{b} w(t) \Delta t} \int_{a}^{b} w(x) f(x) d x\right| \leqslant \frac{M^{2}}{\int_{a}^{b} w(t) \Delta t} \int_{a}^{b}\left(\int_{a}^{b}|(s(x, t))| d t\right) d x .
$$

In Corollary 4.11, if $\mathbb{T}=\mathbb{Z}$, then we have the following result proved in [30].

Corollary 4.13. Let $0<\mathrm{a}<\mathrm{b}, 0 \leqslant \mathrm{k} \leqslant 1, \mathrm{M}=\sup _{\mathrm{a}<\mathrm{n}<\mathrm{b}-1}|\Delta \mathrm{f}(\mathrm{n})|<\infty, \mathrm{N}=\sup _{\mathrm{a}<\mathrm{n}<\mathrm{b}-1}|\Delta \mathrm{f}(\mathrm{n}+1)|<\infty$ and $\mathrm{f}$, $\mathrm{h}:[\mathrm{a}, \mathrm{b}]_{\mathbb{Z}} \rightarrow \mathbb{R}$, such that $\Delta \mathrm{h}(\mathrm{n})=w(\mathrm{n})$ on $[\mathrm{a}, \mathrm{b}]_{\mathbb{Z}}$. Define

$$
s(m, n)= \begin{cases}h(n)-((1-k) h(a)+k h(m)), & a \leqslant n<m-1 \\ h(n)-(k h(m)+(1-k) h(b)), & m \leqslant n \leqslant b-1 .\end{cases}
$$

Then

$$
\begin{aligned}
& \mid(1-k)\left(f^{2}(b)-f^{2}(a)\right)+\left[(k-1) \frac{f(b)-f(a)}{\sum_{n=a}^{b-1} w(n)}+k \frac{f(b+1)-f(a+1)}{\sum_{n=a}^{b-1} w(n)}\right] \sum_{n=a}^{b-1} w(n) f(n+1) \\
& \quad+k(f(a+1) f(b)-f(b+1) f(a))-\frac{f(b)-f(a)}{\sum_{n=a}^{b-1} w(n)} \sum_{n=a}^{b-1} w(n) f(n+2) \mid \\
& \leqslant \frac{M(M+N)}{\sum_{n=a}^{b-1} w(n)} \sum_{m=a}^{b-1}\left(\sum_{n=a}^{b-1}|(s(m, n))|\right) .
\end{aligned}
$$

In Corollary 4.11, if $h(t)=t$, then we have the following result proved in [30].

Corollary 4.14. Let $\mathrm{a}, \mathrm{b} \in \mathbb{T}$ with $\mathrm{a}<\mathrm{b}, 0 \leqslant \mathrm{k} \leqslant 1, \mathrm{M}=\sup _{\mathrm{a}<\mathrm{t}<\mathrm{b}}\left|\mathrm{f}^{\Delta}(\mathrm{t})\right|<\infty, \mathrm{N}=\sup _{\mathrm{a}<\mathrm{t}<\mathrm{b}}\left|\mathrm{f}^{\Delta}(\sigma(\mathrm{t}))\right|<\infty$ and $f:[a, b]_{\mathbb{T}} \rightarrow \mathbb{R}$ be $\Delta$-differentiable functions. Define

$$
s(x, t)= \begin{cases}t-((1-k) a+k x), & a \leqslant t<x \\ t-(k x+(1-k) b), & x \leqslant t \leqslant b\end{cases}
$$

Then

$$
\begin{aligned}
\mid(1-k)\left(f^{2}(b)-f^{2}(a)\right)+\left[(k-1) \frac{f(b)-f(a)}{b-a}+k \frac{f(\sigma(b))-f(\sigma(a))}{b-a}\right] \int_{a}^{b} f(\sigma(t)) \Delta t \\
+k(f(\sigma(a)) f(b)-f(\sigma(b)) f(a))-\frac{f(b)-f(a)}{b-a} \int_{a}^{b} f\left(\sigma^{2}(t)\right) \Delta t \mid \\
\leqslant \frac{M(M+N)}{b-a} \int_{a}^{b}\left[h_{2}((1-k) a+k t, a)+h_{2}(t,(1-k) a+k t)\right. \\
\left.\quad h_{2}(t, k t+(1-k) b)+h_{2}(b, k t+(1-k) b)\right] \Delta t .
\end{aligned}
$$

In Corollary 4.14, if $\mathbb{T}=\mathbb{R}$, then we have the following result proved in [30]. 
Corollary 4.15. Let $0<\mathrm{a}<\mathrm{b}, 0 \leqslant \mathrm{k} \leqslant 1, \mathrm{M}=\sup _{\mathrm{a}<\mathrm{t}<\mathrm{b}}\left|\mathrm{f}^{\prime}(\mathrm{t})\right|<\infty$ and $\mathrm{f}:[\mathrm{a}, \mathrm{b}]_{\mathbb{R}} \rightarrow \mathbb{R}$ are differentiable function. Then

$$
\left|\frac{1}{2}(1-k)\left(f^{2}(b)-f^{2}(a)\right)+(k-1) \frac{f(b)-f(a)}{b-a} \int_{a}^{b} f(t) d t\right| \leqslant \frac{M^{2}}{3}(b-a)^{2}\left(2 k^{2}-2 k+1\right) .
$$
$\left.1\left(\mathrm{a}_{1}\right)\right]$.

In Corollary 4.15, if $k=0$, then we have the following result that has been proved in [35, Theorem Corollary 4.16. Let $0<\mathrm{a}<\mathrm{b}, \mathrm{M}=\sup _{\mathrm{a}<\mathrm{t}<\mathrm{b}}\left|\mathrm{f}^{\prime}(\mathrm{t})\right|<\infty$ and $\mathrm{f}:[\mathrm{a}, \mathrm{b}]_{\mathbb{R}} \rightarrow \mathbb{R}$ be differentiable functions. Then

$$
\left|\frac{1}{2}\left(f^{2}(b)-f^{2}(a)\right)-\frac{f(b)-f(a)}{b-a} \int_{a}^{b} f(t) d t\right| \leqslant \frac{M^{2}}{3}(b-a)^{2} .
$$

In Corollary 4.14, if $\mathbb{T}=\mathbb{Z}$, then we have the following result proved in [30].

Corollary 4.17. Let $0<\mathrm{a}<\mathrm{b}, 0 \leqslant \mathrm{k} \leqslant 1, \mathrm{M}=\sup _{\mathrm{a}<\mathrm{n}<\mathrm{b}-1}|\Delta \mathrm{f}(\mathrm{n})|<\infty, \mathrm{N}=\sup _{\mathrm{a}<\mathrm{n}<\mathrm{b}-1}|\Delta \mathrm{f}(\mathrm{n}+1)|<\infty$ and $f:[a, b]_{\mathbb{Z}} \rightarrow \mathbb{R}$. Then

$$
\begin{aligned}
& \mid(1-k)\left(f^{2}(b)-f^{2}(a)\right)+\left[(k-1) \frac{f(b)-f(a)}{b-a}+k \frac{f(b+1)-f(a+1)}{b-a}\right] \sum_{n=a}^{b-1} f(n+1) \\
& \quad+k(f(a+1) f(b)-f(b+1) f(a))-\frac{f(b)-f(a)}{b-a} \sum_{n=a}^{b-1} f(n+2) \mid \\
& \leqslant \frac{M(M+N)}{3}\left[(b-a)^{2}\left(2 k^{2}-2 k+1\right)+k^{2}-4 k+2\right] .
\end{aligned}
$$

\section{References}

[1] R. P. Agarwal, M. Bohner, A. Peterson, Inequalities on time scales: a survey, Math. Inequal. Appl., 4 (2001), $535-557$. 1

[2] R. P. Agarwal, D. O'Regan, S. H. Saker, Dynamic Inequalities on Time Scales, Springer, Cham, (2014). 1

[3] R. P. Agarwal, D. O’Regan, S. H. Saker, Hardy Type Inequalities on Time Scales, Springer, Cham, (2016). 1

[4] R. P. Agarwal, P. Y. H. Pang, Opial Inequalities with Applications in Differential and Difference Equations, Kluwer Academic Publishers, Dorderchet (1995). 3

[5] G. A. Anastassiou, Duality principle of time scales and inequalities, Appl. Anal., 89 (2010), 1837-1854. 1

[6] N. S. Barnett, S. S. Dragomir, An inequality of Ostrowski's type for cumulative distribution functions, Kyungpook Math. J., 39 (1999), 303-311. 1

[7] N. S. Barnett, S. S. Dragomir, An Ostrowski type inequality for a random variable whose probability density function belongs to $\mathrm{L}_{\infty}[\mathrm{a}, \mathrm{b}]$, Nonlinear Anal. Forum, 5 (2000), 125-135. 1

[8] M. Bohner, T. S. Hassan, T. X. Li, Fite-Hille-Wintner-type oscillation criteria for second-order half-linear dynamic equation with deviating arguments, Indag. Math. (N.S.), 29 (2018), 548-560. 1

[9] M. Bohner, T. X. Li, Kamenev-type criteria for nonlinear damped dynamic equations, Sci. China Math., 58 (2015), 14451452. 1

[10] M. Bohner, T. Matthews, The Grüss inequality on time scales, Commun. Math. Anal., 3 (2007), 1-8. 1

[11] M. Bohner, T. Matthews, Ostrowski inequalities on time scales, JIPAM. J. Inequal. Pure Appl. Math., 9 (2008), 8 pages. $1,1,3,4.1$

[12] M. Bohner, A. Peterson, Dynamic Equations on Time Scales, An Introduction with Applications, Birkhäuser, Boston, (2001). 2, 2

[13] M. Bohner, A. Peterson, Advances in Dynamic Equations on Time Scales, Birkhäuser Boston, Boston, (2003). 2

[14] P. L. ČebyŠev, Sur les expressions approximatives des integrales definies par les autres prises entre les memes limites, Proc. Math. Soc. Charkov, 2 (1882), 93-98. 1

[15] K.-S. Chiu, T. X. Li, Oscillatory and periodic solutions of differential equations with piecewise constant generalized mixed arguments, Math. Nachr., 292 (2019), 2153-2164. 1

[16] S. S. Dragomir, P. Cerone, J. Roumeliotis, A new generalization of Ostrowskis intergral inequality for mappings whose derivatives are bounded and applications in numerical integration and for special means, Appl. Math. Lett., 13 (2000), 19-25. 1 
[17] J. Džurina, S. R. Grace, I. Jadlovská, T. X. Li, Oscillation criteria for second-order Emden-Fowler delay differential equations with a sublinear neutral term, Math. Nachr., 293 (2020), 910-922. 1

[18] S. Frassu, C. van der Mee, G. Viglialoro, Boundedness in a nonlinear attraction-repulsion Keller-Segel system with production and consumption, J. Math. Anal. Appl., 504 (2021), 20 pages. 1

[19] S. Frassu, G. Viglialoro, Boundedness for a fully parabolic Keller-Segel model with sublinear segregation and superlinear aggregation, Acta Appl. Math., 171 (2021), 20 pages. 1

[20] G. Grüss, Über das Maximum des absoluten Betrages von $\frac{1}{(b-a)} \int_{a}^{b} f(x) g(x) d x-\frac{1}{(b-a)^{2}} \int_{a}^{b} f(x) d x \int_{a}^{b} g(x) d x, M a t h$. Z., 39 (1935), 215-226. 1

[21] S. Hilger, Ein Maßkettenkalkül mit Anwendung auf Zentrumsmannigfaltigkeiten, Ph. D. Thesis, Universität Würzburg, (1988). 1

[22] S. Hilger, Analysis on measure chains-A unified approach to continuous and discrete calculus, Result Math., 18 (1990), 18-56.

[23] Z. A. Karian, E. J. Dudewicz, Fitting Statistical Distributions, CRC Press, Boca Raton, (2000). 1

[24] B. Karpuz, U. M. özkan, Generalized Ostrowski's inequality on time scales, JIPAM. J. Inequal. Pure Appl. Math., 9 (2008), 7 pages. 1,1

[25] T. X. Li, Y. V. Rogovchenko, On the asymptotic behavior of solutions to a class of third-order nonlinear neutral differential equations, Appl. Math. Lett., 105 (2020), 7 pages. 1

[26] T. Li, G. Viglialoro, Boundedness for a nonlocal reaction chemotaxis model even in the attraction dominated regime, Differ. Integ. Equ., 34 (2021), 315-336. 1

[27] W.-J. Liu, Some weighted integral inequalities with a parameter and application, Acta Appl. Math., 109 (2010), 389-400. 1,3

[28] W. J. Liu, Q.-A. Ngô, W. B. Chen, A new generalization of Ostrowski type inequality on time scales, An. Ştiinţ. Univ. "Ovidius" Constanţa Ser. Mat., 17 (2009), 101-114. 1, 1

[29] T. X. Li, N. Pintus, G. Viglialoro, Properties of solutions to porous medium problems with different sources and boundary conditions, Z. Angew. Math. Phys., 70 (2019), 18 pages. 1

[30] W. J. Liu, A. Tuna, Weighted Ostrowski, trapezoid and Grüss type inequalities on time scales, J. Math. Inequal., 6 (2012), 381-399. 1, 1, 3, 3, 3, 3, 3, 4.1, 4.1, 4.1, 4.1, 4.1, 4.1, 4.1, 4.2, 4.2, 4.2, 4.2, 4.2, 4.2

[31] D. S. Mitrinović, J. E. Pečarić, A. M. Fink, Inequalities involving function and their integrals and derivatives, Kluwer Acad. Publ., Dordrecht, (1991). 1

[32] D. S. Mitrinović, J. E. Peĉarić, A. M. Fink, Inequalities Involving Functions and Their Integrals and Derivatives, Kluwer Academic Publishers, Dordrecht, (1991). 1

[33] Q.-A. Ngô, W. J. Liu, A sharp Grüss type inequality on time scales and application to the sharp Ostrowski-Grüss inequality, Commun. Math. Anal., 6 (2009), 33-41.

[34] A. Ostrowski, Uber die absolutabweichung einer differentierbaren Funktion von ihren Integralmittelwert, Comment. Math. Helv., 10 (1938), 226-227. 1

[35] B. G. Pachpatte, On trapezoid and Grüss-like integral inequalities, Tamkang J. Math., 34 (2003), 365-369. 1, 4.2

[36] S. H. Saker, Hardy-Leindler type inequalities on time scales, Appl. Math. Inf. Sci., 8 (2014), 2975-2981. 1

[37] S. H. Saker, R. R. Mahmoud, M. M. Osman, R. P. Agarwal, Some new generalized forms of Hardy's type inequality on time scales, Math. Inequal. Appl., 20 (2017), 459-481.

[38] S. H. Saker, R. R. Mahmoud, A. Peterson, Some Bennett-Copson type inequalities on time scales, J. Math. Inequal., 10 (2016), 471-489. 1

[39] S. H. Saker, R. R. Mahmoud, A. Peterson, Weighted Hardy-type inequalities on time scales with Applications, Mediter. J. Math., 13 (2016), 585-606.

[40] S. H. Saker, D. O’Regan, Hardy and Littlewood inequalities on time scales, Bull. Malays. Math. Sci. Soc., 39 (2016), 527-543. 1

[41] S. H. Saker, D. O’Regan, R. P. Agarwal, Generalized Hardy, Copson, Leindler and Bennett inequalities on time scales, Math. Nachr., 287 (2014), 686-698.

[42] S. H. Saker, M. M. Osman, D. O’Regan, R. P. Agarwal, Some new Opial dynamic inequalities with weighted functions on time scales, Math. Inequal. Appl., 18 (2015), 1171-1187. 1

[43] S. H. Saker, M. M. Osman, D. O’Regan, R. P. Agarwal, Inequalities of Hardy-type and generalizations on time scales, Analysis (Berlin), 38 (2018), 47-62.

[44] S. H. Saker, M. M. Osman, D. O’Regan, R. P. Agarwal, Lyapunov inequalities for dynamic equations via new Opial type inequalities, Hacettepe J. Math. Stat., 47 (2018), 1544-1558.

[45] S. H. Saker, M. M. Osman, D. O'Regan, R. P. Agarwal, Levinson type inequalities and their extensions via convexity on time scales, Rev. R. Acad. Cienc. Exactas Fís. Nat. Ser. A Mat. RACSAM, 113 (2019), 299-314. 1

[46] A. Tuna, D. Daghan, Generalization of Ostrowski and Ostrowski-Grüss type inequalities on time scales, Comput. Math. Appl., 60 (2010), 803-811. 1, 3

[47] S. F. Wang, Q. L. Xue, W. J. Liu, Further generalization of Ostrowski-Grüss type inequalities, Adv. Appl. Math. Anal., 3 (2008), 17-20. 